\title{
Philosophiques
}

\section{La méthode du discours : la philosophie}

\section{Michel Guérin}

Volume 22, numéro 2, automne 1995

URI : https://id.erudit.org/iderudit/027331ar

DOI : https://doi.org/10.7202/027331ar

Aller au sommaire du numéro

Éditeur(s)

Société de philosophie du Québec

ISSN

0316-2923 (imprimé)

1492-1391 (numérique)

Découvrir la revue

Citer cet article

Guérin, M. (1995). La méthode du discours : la philosophie. Philosophiques, 22(2), 247-263. https://doi.org/10.7202/027331ar

\section{Résumé de l'article}

$\mathrm{Au}$ premier degré, cette étude se lit comme une réflexion sur le concept de méthode. L'examen des textes cartésiens permet de dégager les caractères de la méthode (comme Vordre volontaire, la médiation, la réflexivité), mais surtout ce qu'on peut appeler son " esprit ». Il tient tout entier, semble-t-il, dans la découverte - qui vaut invention - de la fécondité du principe : la pensée élaborée, en effet, aperçoit d'un même mouvement l'homogénéité du principe et de la fin, d'une part, l'homologie entre la pensée et l'Être, d'autre part. À un niveau plus profond, la transcendance de la méthode par rapport à ses règles (d'où le renversement du discours de la méthode en méthode du discours) conduit, dans un premier temps, à l'identifier avec la philosophie elle-même appréhendée ici comme « commentaire de son principe " - dans un second temps à marquer, sous le rapport du modèle, la différence entre la philosophie et les sciences.
Ce document est protégé par la loi sur le droit d'auteur. L'utilisation des services d’Érudit (y compris la reproduction) est assujettie à sa politique d'utilisation que vous pouvez consulter en ligne.

https://apropos.erudit.org/fr/usagers/politique-dutilisation/ 


\title{
LA MÉTHODE DU DISCOURS : LA PHILOSOPHIE
}

\author{
par \\ Michel Guérin
}

RÉSUMÉ : Au premier degré, cette étude se lit comme une réflexion sur le concept de méthode. L'examen des textes cartésiens permet de dégager les caractères de la méthode (comme l'ordre volontaire, la médiation, la réflexivité), mais surtout ce qu'on peut appeler son " esprit». Il tient tout entier, semble-t-il, dans la découverte - qui vaut inventionde la fécondité du principe: la pensée élaborée, en effet, aperçoit d'un même mouvement l'homogénéité du principe et de la fin, d'une part, l'homologie entre la pensée et l'Être, d'autre part.

$\grave{A}$ un niveau plus profond, la transcendance de la méthode par rapport à ses règles (d'où le renversement du discours de la méthode en méthode du discours) conduit, dans un premier temps, à l'identifier avec la philosophie elle-mêmeappréhendée ici comme "commentaire de son principe" dans un second temps à marquer, sous le rapport du modèle, la différence entre la philosophie et les sciences.

ABSTRACT: As far as the literal meaning is concerned, this study can be read as a reflection on the concept of the method. The perusal of Descartes's texts allows to bring out the characteristics of the method (such as voluntary order, mediation, reflexibility), but especially what may be called its "spirit". It entirely lies, so it seems, in the discoverywhich is as good as invention - of the potential of the principle : indeed, elaborate thought straightaway perceives the homogeneity of the principle and of the end, on the one hand, the homology between thought and Being, on the other hand. As far as the hidden meaning is concerned, the transcendence of the method with regard to its rules (from which the reversal of a discourse on method into a method on discourse) leads first to identify it with philosophy itself - in this instance considered as a "commentary on its principle " - subsequently to lay stress, from the point of view of the model, on the difference between philosophy and sciences. 
À tout seigneur tout honneur, et puisque je prends ici le parti de renverser le discours de la méthode en méthode du discours, entrons donc dans le sujet par Descartes. Alain remarque « que l'idee d'ordre est partout dans Descartes, dont la pensée la plus familière est qu'avant de connaître une chose, il faut en connaître d'abord plusieurs autres $»$, puisqu'aussi bien, continue-t-il, tel est «ce mouvement de l'esprit qui toujours dépasse ${ }^{1}$ ». Le premier effet de la méthode est ainsi d'éloigner de notre portée les objets naturels, à la lettre de les déporter, ou, comme dit encore Alain, de " refuser d'abord cette nature, qui nous jette tout à la fois $»$. Bref, la méthode commence par l'écart, puisque ce n'est qu'en renonçant délibérément à l'objet immédiat qu'elle se promet l'objet pensé à sa place parmi d'autres. Sous ce rapport, la méthode désignerait la pensée considérée dans sa vertu médiatrice. En s'interdisant la première prise, dans ce qu'elle peut avoir à la fois d'atomique et de chaotique, d'épisodique et de holistique, la pensée n'assure-t-elle pas, en la différant, son emprise sur ses objets? Mais comment comprendre l'articulation de cette retraite, d'ailleurs tactique, avec la stratégie d'anticipation que la méthode met en œuvre ? Ouvrons la problématique par cette question, introduisant à une sorte de phénoménologie de la méthode.

\section{L'esprit de suite}

L'histoire de la philosophie comme l'histoire des sciences nous ont légué des «méthodes » et des discours dessus qui ont fait date. Il est trop clair que nous n'avons ici ni l'illusoire prétention de vérifier les titres, ni de constituer, comme en surplomb, une méthode des méthodes. Et à celui qui entendrait tenter une histoire du concept de méthode, comme si le mot était resté stable, les choses seules changeant ou plutôt se succédant sous l'affiche, nous ne pourrions que demander : selon quelle méthode allez-vous vous orienter dans cette forêt, ou, pour reprendre Descartes en sa Règle V des Regula, par quel fil de Thésée pénétrerez-vous dans le labyrinthe ?

Nous donnerons donc l'exemple de la méthode par un premier mouvement de retraite devant tant de pensées consignées et homologuées en choisissant, dans un esprit cartésien aussi, de partir du plus simple. Sans certitude de détecter l'essence de la méthode, nous essaierons au moins d'en saisir un peu l'esprit et, abdiquant la connaissance, nous nous contenterons de reconnaître, s'il se peut, quelques signes qui ne trompent pas.

Le premier, Alain a mis le doigt dessus, c'est un ordre qui provient d'un désinvestissement médité du monde naturel. Le mouvement par quoi l'ordre se profile est concomitant d'une dénégation des objets particuliers. Simplement dit : la connaissance ne peut commencer et faire valoir son intérêt propre que lorsque je cesse de m' intéresser immédiatement à cet objet-ci. La pensée a besoin de penser qu'elle se déprend d'une obnubilation première, quelque

1. Alain, « Descartes » in Les Passions et la Sagesse, Paris, Gallimard, p. 968. 
nom qu'elle donne à cette glu ; c'est le propre de toutes les pensées élaborées, qu'elles se donnent comme sciences ou philosophies, que de marquer, en leur pli même, la mémoire d'un détachement. Dans son écriture même, à cet égard, le Discours de la méthode demeure entre tous l'exemple.

Demeurant " tout le jour enfermé seul dans un poêle " alors qu'au-dehors sévissent l'hiver (1619-1620) et la guerre (de Trente Ans), Descartes se retire en lui-même, ou plutôt se ramène à lui-même et fait retour sur soi : « Jamais, écrit-il, mon dessein ne s'est étendu plus avant que de tâcher à réformer mes propres pensées, et de bâtir dans un fonds qui est tout à $\mathrm{moi}^{2}$. ». Comme on voit d'abord, ordre s'entend de deux façons : c'est celui qu'on se donne, c'est encore celui qu'on met dans ses pensées. On ne sait bien régir que son propre fonds. L'idée d'ordre enveloppe ici la réduction au propre; elle s'atteste de la butée sur soi. À la lettre, la méthode est une pensée appropriée.

Son mode, dès lors, ne saurait être que la réflexivité. La décision de penser par soi et la distance prise par rapport au donné (le monde naturel) ou au reçu (les opinions, les livres, ce qu'on appellerait aujourd'hui l'héritage culturel) placent nécessairement l'entreprise, c'est-à-dire l'effort ou le courage de penser « tâcher à "...) sur le plan de la réflexion. Une méthode non-réflexive apparaît comme une contradiction dans les termes. Si le signe sûr d'une pensée réfléchie est la conscience de son origine comme effort, la méthode ne s'invente qu'en se découvrant concomitamment volonté conséquente. Autant dire qu'elle s'impose à soi ensemble comme principe et comme fin. En même temps qu'elle est, par essence, une pensée des commencements, la méthode se déploie comme pensée finale, par quoi s'entend moins une pensée des fins qu'une démarche intellectuelle qui ne s'arrêtera qu'à la fin.

En ce point aussi, en dépit ou à cause de ses prétéritions répétées (de ne conseiller que $\mathrm{soi}^{3}$ ), Descartes fait exemple.

C'est, en effet, qu'il fait sortir la portée de la méthode du principe où elle se fonde et d'abord, tout court, du principe comme tel. Le premier mérite d'une méthode découle plus de la vigueur du principe que de sa teneur particulière. En d'autres termes, la méthode est une illustration du principe comme principe. La force du principe, Descartes l'appréhende en tant que nécessité de la méthode ${ }^{4}$ et suggère que des « vérités » obtenues par chance ou rencontrées par hasard ne méritent pas ce nom. Quant à ce qu'on pourrait appeler l'ampleur de la méthode, elle se tire de la nécessité, dans la mesure où celle-ci instaure l'unité du savoir. Il s'agit bien d'unité, et non de totalité ; j'y reviendrai ultérieurement. Une définition possible de la méthode pourrait

2. René Descartes, Discours de la méthode, Deuxième partie, Paris, Gallimard, p. 135.

3. «Ainsi mon dessein n'est pas d'enseigner ici la méthode que chacun doit suivre pour bien conduire sa raison, mais seulement de faire voir en quelle sorte j'ai tâché de conduire la mienne. » (op.cit., Première partie, p. 127.)

4. René Descartes, Règles pour la direction de l'esprit, Règle IV, La méthode est nécessaire pour la recherche de la vérité. 
être : la fécondité du principe (Descartes parle de "fruits légitimes » ou d' « espérance »). Dès qu'elle se saisit comme mode nécessaire et voie unique de la pensée qui progresse, la méthode se convainc qu'elle porte loin, qu'elle s'applique sur toute la largeur du savoir. Dans l'esprit du Livre VI de la République et de son héliotropisme ${ }^{5}$, ne voit-on pas Descartes affirmer, en ouverture de ses Règles pour la direction de l'esprif́, que "si quelqu'un veut chercher sérieusement la vérité, il ne doit donc pas choisir l'étude de quelque science particulière : car elles sont toutes unies entre elles et dépendent les unes des autres; mais il ne doit songer qu'à accrôtre la lumière naturelle de sa raison"?

Si volontaire qu'elle soit, la méthode ne saurait être arbitraire, sauf à devoir constater rapidement son impasse. Mais, inversement, si le cheminement de l'esprit se règle sur la nature des choses, la méthode est-elle encore quelque chose par elle-même ? Trop coupée de l'Être, elle court le risque de stérilité ; strictement commandée par lui, ne médite-t-elle pas sa propre exténuation, sinon disparition? À moins, justement, de faire de la méthode ellemême le carrefour où se croisent et se nouent l'Être et la pensée. La solution platonicienne - pour le dire vite - consiste à ériger le Bien en principe qui, comme traduit Léon Robin, « aux objets de connaissance procure la réalité et qui confere au sujet connaissant le pouvoir de connaître » (508e). "Cause du savoir et de la réalité ", le Bien aimante la connaissance en lui prêtant, pour ainsi dire, sa réalité. Mais si la méthode - en l'espèce la dialectique parvient ainsi à joindre les plans gnoséologique et ontologique, c'est à une double condition : dans l'ordre de l'Etre, la transcendance du Bien qui, dit Glaucon, "surpasse en beauté » (509a) réalité et savoir que, dans le même temps, il fonde; dans l'ordre du savoir, l'exigence d'avancer jusqu'à l'anhypothétique au lieu de se contenter, à l'instar du discours mathématique, de raisonner à l'intérieur d'hypothèses. Du coup, la méthode dialectique correspond à l'Être, mais ne le réfléchit pas passivement, justement parce qu'il est transcendance et qu'il se donne en se retirant comme pure différence. C'est parce qu'elle se fonde dans l'Être que la méthode est fructueuse; c'est parce que l'Être est différence qu'elle doit chercher en son propre fonds la ressource de le suivre.

À la lettre, donc, la méthode enseigne une douce violence, puisque, selon la proposition fameuse, «ce n'est pas assez d'avoir l'esprit bon, mais le

5. Platon, République, VI, 508.

6. René Descartes, $O p$. cit., Règle I. En particulier : «Car, étant donné que toutes les sciences ne sont rien d'autre que la sagesse humaine, qui demeure toujours une et toujours la même, si différents que soient les objets auxquels elle s'applique, et qui ne reçoit pas plus de changement de ces objets que la lumière du soleil de la variété des choses qu'elle éclaire, il n'est pas besoin d'imposer de bornes à l'esprit : la connaissance d'une vérité ne nous empêche pas en effet d'en découvrir une autre, comme l'exercice d'un art nous empêche d'en apprendre un autre, mais bien plutôt elle nous y aide " (p. 37-38). 
principal est de l'appliquer bien ${ }^{7}$ ». En même temps qu'il se fie à « la lumière de l'esprit ", " ces premières semences de vérité, déposées par la nature dans l'esprit humain ${ }^{8}$ », Descartes, c'est le cas de le dire, rappelle l'esprit à l'ordre. À la douceur séminale s'oppose l'armement de l'esprit, le mot s'entendant à la fois comme le gréement d'un navire à l'appareillage et comme le fourbir militaire. La méthode apparaît ainsi comme le chiasme d'une confiance ontologique et d'un volontarisme, voire d'un militantisme gnoséologique. Et c'est bien, toujours, dans cet esprit, qu'un Heidegger peut parler de la « douce rigueur » et de la « rigoureuse douceur du laisser-être de l'étant comme tel en totalite $e^{9}$ ". Aussi bien, ce qui est ici en jeu, et qu'on réserve pour un examen ultérieur, c'est, pour choisir, au positif, un mot de Rousseau, l' « activité » de la méthode, ou plutôt son degré d'activité (l'auteur d'Émile caractérisant, comme on le sait, la sienne comme « inactive »)... A priori toutefois, ne peuton pas supposer que, plus la méthode est active, plus elle confisque l'Être au profit de sa propre veine représentative, tandis qu'à l'autre extrémité ce serait précisément d'une « méthode inactive » que de le laisser parler tout seul et comme naturellement?

Quoi qu'il en soit, il suffit au profil qu'on essaie d'abord de dessiner de marquer, au cour de toute méthode, la jointure du « naturel » et de l' « artificiel ». Paradigmatique toujours, Descartes souligne également les deux traits, et à travers le concept d'ordre lui-même, lequel, comme l'a bien vu Alain, n'a son sens que de composer le « naturel » et ce qui l'est moins. En effet, s'il est dans la nature des choses de commencer par l'intuition du simple, la déduction qui sensuit est plus ou moins à la discrétion de l'esprit en son agir ; et c'est tout l'esprit de la proposition cartésienne ( $3^{\mathrm{e}}$ règle de la Méthode), de supposer « même de l'ordre » entre les objets « qui ne se précèdent point naturellement les uns les autres ${ }^{10}$ ». D'où, de l'aveu même de Descartes, l'anodine importance de la Règle VI, qui paraît « rien apprendre de bien nouveau ", tandis qu'elle « contient le principal secret de la méthode ». "Elle enseigne en effet que les choses peuvent être rangées en différentes séries ${ }^{11}$ ». Le « secret » de la méthode, c'est la « série ». L'ordre est naturel parce qu'il part du simple, c'est-à-dire de l'absolu ; il est « art », par un autre aspect, puisqu'il consiste à disposer les choses en séries. Or, si notre hypothèse est juste, d'une mixité de la méthode, apparentée à l'Être et au connaître, celle-ci ne se donne pas comme une suite "naturelle », mais s'atteste en tant qu'esprit de suite, pour-suite.

7. René Descartes, Discours de la méthode, p. 126.

8. René Descartes, Règles pour la direction de l'esprit, Règle IV, p. 49.

9. Martin Heidegger, "De l'essence de la vérité », in Question I, Gallimard, p. 190.

10. René Descartes, Discours de la méthode, Deuxième partie, Gallimard, p. 138.

11. René Descartes, Règles pour la direction de l'esprit, Règle VI, Gallimard, p. 53. 


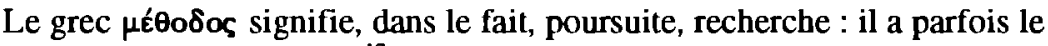

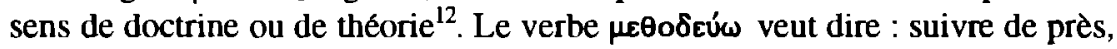
à la piste, régulièrement ; poursuivre, rechercher. Suivre la piste ou encore le $f i l$, voilà l'essence de la méthode. Sous cet angle, on le remarquera, apparaissent clairement et ce que $\mathrm{j}$ 'appellerais la rémanence de l'Être (sa trace, son sillage) et la résolution d'avancer sur le chemin qui s'ouvre, c'est-à-dire, strictement, de pro-céder. Il n'y aurait pas de méthode, du moins dans l'acception philosophique du terme, si l'Être ne pouvait être suivi à la trace, s'il ne nous mettait lui-même sur la piste. Et même si l' itinerarium mentis in veritatem n'est pas toujours droit selon l'image, empiriquement ; même s'il connaît l'empêchement, l'arrêt prolongé, le ralentissement, la diversion, voire l'errance $^{13}$, il n'en demeure pas moins - transcendantalement, si l'on peut dire - le « droit chemin "; même s'il n'est pas rectiligne, il conserve sa rectitude, il ne se perd pas lui-même ; il porte son esprit avec soi, sa persévérance. À cet égard, du " plus long détour " platonicien aux "Holzwege " heideggériens, en passant par le « droit chemin » cartésien et le « kritische Weg » kantien, la conséquence est bonne.

Récapitulons. La méthode est médiation, passage de l'esprit naturel à l'entendement conscient de sa charge. Elle se donne comme convenant, éminemment, à la raison qui la cultive et qui, s'y singularisant, en peut faire valoir l'universalité ou, à l'instar de Descartes précisément, en faire discours. Réflexive nécessairement, elle est volontaire sans être arbitraire, dans la mesure où elle découvre, par une sorte de réfraction de la fin dans le principe, que la voie à suivre traverse l'unité du savoir et non pas telle région particulière. Tout en s'éprouvant comme effort, voire comme essai, elle se conforte d'une manière de foi ontologique, qui lui donne à penser que le travail à quoi elle s'astreint transforme l'offre initiale de l'Être. Enfin et corollairement, la méthode s'affirme dynamiquement comme esprit de suite, poursuite.

Tel est, croyons-nous, l'esprit de la méthode. Nous réservant de dire un mot, un peu plus tard, de la différence entre une méthode scientifique (ou se voulant telle) et la méthode philosophique (ou plutôt la philosophie comme méthode), nous devons à présent faire droit à la question : la méthode ne désigne-t-elle que l'esprit de la recherche, fait-elle à ce point corps avec sa mise en ouvre si bien qu'elle est indiscernable en tant qu'objet de savoir, ou bien au contraire, peut-on en faire thème et l'anatomiser comme paraît l'avoir fait, en son fameux Discours, Descartes le premier?

12. Platon, Théétète, $189 \mathrm{c}$ (il est question de la " doctrine, d'après laquelle tout se meurt »).

13. Goethe, dans le Premier Faust : « Es irrt der Mensch, solange er strebt ». 


\section{DU DISCOURS DE LA MÉTHODE}

Reprenons une fois de plus le modèle cartésien, soit le Discours de la méthode. En première analyse, nous aurions tout lieu de conclure que la méthode est l'objet du discours du même nom. Nous pouvons, en effet, le thématiser exhaustivement et le ramener à l'ensemble des préceptes et des maximes nettement identifiés par l'auteur. En seconde analyse, toutefois, les choses ne paraissent plus aussi simples. D'abord parce que nous ne saurions négliger un double avertissement de Descartes. Le premier (nous nous y sommes déjà arrêtés), c'est l'insistance à rapporter la méthode à son seul auteur : si cette sorte d'abdication de prétention ne vient ni de prudence ni de coquetterie, que veut-elle dire à la fin ? N'est-elle pas, d'ailleurs, en contradiction avec le titre et, pour ainsi dire, le régime même du « discours », lequel impose publication, diffusion et persuasion universelle ? N'y a-t-il pas quelque contrariété dans un propos qui, en même temps qu'il se veut prouver (par quoi il en appelle à tous les hommes et à la raison humaine comme telle), fait tout ce qu'il faut pour se dédire et n'a l'air d'afficher une ambition que pour en réduire à l'extrême le champ d'exercice ( «tâcher à réformer mes propres pensées ») ? Le deuxième avertissement est plus sérieux encore : il contredit pourtant apparemment le premier en un point, car, en même temps qu'il annonce ce que j'appellerais je vais m'en expliquer - la déception objective de la méthode, il suppose que, de tout temps, elle a été connue, sinon reconnue, par « les plus grands esprits ". D'un côté, donc, Descartes répète : la méthode est mienne; de l'autre côté, il allègue "les principes innés de cette méthode ${ }^{14}$ », tels qu'ils n'ont pas pu ne pas être familiers aux meilleurs esprits « depuis longtemps ». Ainsi, de deux choses l'une : soit la méthode est un « art " nouveau, et il en faut rapporter le mérite à son auteur ; soit tout esprit qui, par le passé, a pensé un peu fermement, en a eu, comme dit le texte "quelque idée »; et alors, en perdant ses caractères inédit et personnel, la méthode se résorbe dans la pensée philosophique, celle, précisément, de ces « grands esprits» qui se sont affrontés aux questions dernières de la pensée. La méthode est donc appréhendée par Descartes selon une double perspective : comme art personnel, elle ne veut que la réforme de l'entendement de celui qui l'invente ; sous le rapport, maintenant, de sa quasi-innéité, elle finit par se calquer sur le conatus de l'esprit humain, pourvu que, même occupé de problèmes déterminés, il soit mu par un intérêt universel touchant le savoir comme tel. La méthode est donc saisie à travers sa foncière ambiguité, s'il est vrai qu'elle se donne à la fois comme thème insistant du discours et comme rémanence d'une histoire de la pensée ${ }^{15}$. Ensemble actuelle et passée, ou plutôt urgente et immémoriale, on

14. René Descartes, Rèyles pour la direction de l'esprit, Règle IV, p. 47.

15. Si éloigné que soit Descartes de l'idiosyncrasie historisante, c'est, on le rappellera, comme « une histoire, ou, si vous l'aimez mieux... comme une fable » qu'il propose par écrit son « exemple » dans le Discours de la méthode, (Première partie, p. 127). 
dirait qu'elle ne s'explicite que pour retrouver son fantôme spontané. Elle se définirait, d'un oxymoron : une invention naturelle.

De là ce que $j$ 'appelais l'effet de déception objective, c'est-à-dire cette énigmatique complétude qui introduit comme un hiatus entre le dire et le faire, la règle et son application et confere, en somme, au programme même d'un « discours de la méthode " une connotation de paradoxe. Écoutons Descartes en cette décisive Règle IV : " Mais si la méthode explique bien comment il faut se servir de l'intuition, pour ne pas tomber dans l'erreur contraire à la vérité, et comment il faut faire des déductions, pour parvenir à la connaissance de toutes choses, rien d'autre n'en est requis, me semble-t-il, pour qu'elle soit complète, puisqu'aucune science ne peut exister, ainsi qu'il a déjà été dit, si ce n'est par intuition ou par déduction. Elle ne peut aller en effet jusqu'à enseigner aussi comment ces opérations mêmes doivent être faites, car elles sont les plus simples et les premières de toutes, en sorte que, si notre entendement ne pouvait déjà les faire auparavant, il ne comprendrait aucun des préceptes de la méthode elle-même, si faciles qu'ils soient ${ }^{16}$. "Comme on voit, l'interprétation du sens de la méthode, loin de passer par le « de deux choses l'une " que nous mentionnions d'abord, exige bien plutôt les deux choses ensemble : en l'espèce, pour reprendre les mots mêmes de Descartes, elle " explique " un comment (comment il faut se servir de l'intuition... comment il faut faire des déductions »), mais ne peut «enseigner » un autre comment ( " comment ces opérations mêmes doivent être faites"). Bien plus, la compréhension de la méthode se suppose elle-même, s'induit à partir d'une pratique première. Bref, la méthode implique une précompréhension de la méthode. Et c'est, au bout du compte, sa dimension originelle qui empêche qu'elle soit originale ou, en d'autres mots, c'est son authenticité qui fait obstacle à sa nouveauté, en même temps, d'ailleurs, qu'à son anatomisation objective ; car si la méthode se pouvait ramener à des procédés techniques particuliers, ce serait au détriment de ce qu'elle a de « naturel », autant dire de son universalité. Descartes souligne d'une part que l'« esprit humain possède en effet je ne sais quoi de divin, où les premières semences de pensées utiles ont été jetées ${ }^{17}$ "; d'autre part que, si les mathématiques foumissent un modèle privilégié de la méthode, il ne faut pas s'arrêter aux « mathématiques ordinaires ", mais chercher la « vraie mathématique » - qui, précise-t-il, étymologiquement «signifie simplement science »-, ou encore la «mathématique universelle ${ }^{18} »$. En d'autres termes, la méthode renvoie, non pas à une pragmatique, mais à une mathesis. Elle est reliée principiellement au projet d'une science universelle. Cette méta-mathématique ou « mathématique vraie» est aussi une méta-physique en ce qu'elle touche purement "l'ordre et la mesure

16. Ibid., p. 47

17. Ibid, p. 51

18. Ibid., p. 51. 
sans application à une matière particulière ${ }^{19} "$, en ce qu' elle vise l'interprétation de l'Être comme tel dans son ultime simplicité. Nous croyons, à cet égard, que, sans nier, naturellement, les différences, il existe une analogie de démarche et de visée entre le Livre VI de la République et cette Règle IV et que, mutatis mutandis, la " mathématique vraie " est dans le même rapport de transcendance aux «mathématiques ordinaires » que la contemplation ou l'intellection dialectique à la démonstration. De même que, chez Platon, la dialectique ne révèle sa véritable portée méthodologique qu'en rabattant le discursif sur les hypothèses qui le cernent, de la même façon, la méthodemathesis, connaissance du simple et du premier, a besoin du discours, y compris dans l'acception rhétorique du mot, pour laisser transparaître en quoi elle l'excède, c'est-à-dire en quoi, tout au rebours, elle est, radicalement, méthode du discours.

Que si, pourtant, l'on objecte que la méthode comporte des règles précises et que, surtout, elle fait, à cet égard, ressortir une distinction entre les préceptes de la science et les maximes de la morale, nous demandons qu'on y regarde d'un peu plus près et que, par-delà l'application, on en restitue l'inspiration, fût-ce au travers de sa vertu de transformation d'un plan à l'autre. Sur l'un et sur l'autre, est à l'ceuvre le même bon sens (cette bona mens divinement ensemencée), quand même il se règle ici sur l'évidence c'est-à-dire sur le certain - et là sur la modération, autant dire sur le probable $^{20}$. À l'évidence de l'idée claire et distincte pour la science, correspond moralement la "réception" de plein coeur de la religion chrétienne et de tout ce qui s'ensuit. Au précepte de la division (de la difficulté) fait écho la résolution de l'esprit ferme ; l'ordre, en troisième lieu, est d'aller du simple au complexe pour connaître ; il est d'accepter celui du monde pour me « rendre content »; enfin, la revue des «diverses occupations qu'ont les hommes » est aussi utile que les « dénombrements si entiers » qui évitent que la chaîne des raisons soit rompue. Or, le domaine de l'agir, qui reprend à son compte les mêmes règles en les transformant, laisse paraître une manière de passivité, en ce sens qu'il subordonne le vouloir, non plus au connu, mais au "reçu» (la religion chrétienne, les lois et coutumes de mon pays, l'ordre du monde); il n'en reste pas moins que la méthode est la même qui, là commande et ici recommande. Il y a d'abord le donné, l'origine (intuition ou révélation, en ôtant de ce dernier mot sa connotation mystique, en lui donnant une valeur «doxique », que rendrait sans doute mieux le mot « réception ») ; puis la résolution humaine : l'analyse qui se garde de la précipitation et de la prévention et la fermeté, qui préserve des "repentirs et remords "; troisièmement, l'interprétation sérielle comme ordre et enfin la synthèse mnémique

19. Ibid., p. 51 .

20. «C'est une vérité très certaine que, lorsqu'il n'est pas en notre pouvoir de discerner les plus vraies opinions, nous devons suivre les plus probables » René Descartes, Discours de la méthode, Troisième partie, p. 142. 
(énumération, revue) qui, dans une région, rend possible la cohérence d'un discours et, dans l'autre, celle d'une conduite.

Dira-t-on, alors, qu'on a affaire à un système ? Dans la rigueur des mots et sous ce rapport précis d'appréhension de la pensée cartésienne, la réponse est négative. Car le système est une totalité synthétique, un ensemble ; le grec

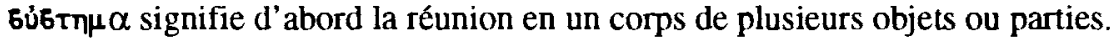
La méthơde n'est pas un système, même si, à côté, elle n'empêche pas qu'un système se présente et s'organise. C'est que la méthode est nécessairement analytique, puisque c'est un mouvement, un chemin. C'est pourquoi, plutôh elle revêt une dimension « pédagogique », non seulement parce qu'elle peut se montrer, mais surtout parce qu'elle est la pensée s'enseignant elle-même, accédant à sa propre conviction en déférant aux obligations de sa tâche. Et c'est bien parce que, radicalement, la méthode est enseignement qu'elle transcende ses règles et que, dans le temps qu'elle les expose, elle les reconduit au principe séminal, à l'engendrement authentique du discours.

La méthode est un déploiement du bon sens ; elle explique ce qu'il enveloppe et ne l'appelle, en fin de compte, qu'à être attentif à soi. C'est pourquoi elle est à la fois peu et beaucoup. Tant et si bien qu' on pourrait exprimer ainsi le paradoxe de la meilleure méthode : celle dont on saurait se passer - à condition de faire bien attention à ce qu'on sait déjà, sans bien savoir qu'on le sait. Si notre intuition est juste, c'est d'ailleurs de la méthode comme philosophie qu'il s'agit alors (et l'on pense ici au Menon), la voie de la pensée ne faisant qu'un avec son auvre ou, pour user du fameux diptyque heideggérien, les Wege se substituant entierement aux Werke ${ }^{21}$.

Il y a un autre " discours de la méthode " qui exhibe ce paradoxe, et c'est croyons-nous, Émile. Explicite ou implicite, la référence cartésienne y est, d'ailleurs, permanente. Bien entendu, le rapprochement n'est pas de doctrine (puisque, pour Rousseau, ce sont les sens qui sont innés, non les idées), mais de forme ; comme en témoigne ce texte, presque un pastiche de la manière cartésienne : "J'ai transcrit cet écrit - la profession de foi du vicaire savoyard - non comme une règle des sentiments qu'on doit suivre en matière de religion, mais comme un exemple de la manière dont on peut raisonner avec son élève pour ne point s'écarter de la méthode que j'ai tâché d'établir $^{2}{ }^{2}$. Et, dans le fait, tout le début de la Profession de foi, au Livre IV d'Émile, démarque la première partie du Discours de la méthode $e^{23}$.

21. Au seuil de l'édition des auvres complètes de Heidegger sont inscrits les mots : «Wege, nicht Werke» (Des chemins, non des ceuvres).

22. Jean-Jacques Rousseau, Emile, Livre IV, Paris, Gallimard, t. IV, p. 635.

23. On rappellera brièvement la modeste protestation initiale de «bon sens » (opposé aux « discours savants » et aux «profonds raisonnements»), la conviction que * la raison nous est commune » et la détermination de penser par soi après constat que les « livres » et les « diverses opinions » des philosophes conduisent à déception. « J'étais, dit le Vicaire, dans ces dispositions d'incertitude que Descartes exige pour la recherche de la vérité » (ibid., p. 567). En somme, le tour d'horizon 
Le temps nous manque ici pour développer comme il le faudrait le parallèle Descartes-Rousseau. Mais il est certain que, si Émile (avec son satellite de circonstance, soit La Lettre à Christophe de Beaumont̀ contient la métaphysique de Rousseau, celle-ci se fonde sur le « j'existe et $\mathrm{j}$ 'ai des sens » avec le même degré de radicalité que celle de Descartes sur le « je suis une chose qui pense ». L'éducation est la forme de la pensée de Rousseau, c'est-à-dire sa méthode en tant qu'elle fait corps avec son principe, qui est, Rousseau y insiste, moins d'enseigner des vérités que d'épargner les erreurs ${ }^{24}$, parce qu'il n'y a rien de vrai en dehors ou à côté de la nature. C'est donc à elle à paraître et à parler et le précepteur, «ministre de la nature », est tout sauf son parasite ou son bruyant interprète; son art est entier d'effacement ${ }^{25}$. Telle est la «méthode inactive ${ }^{26} »$.

Chez Rousseau, le bon sens est comme indexé sur la "bonté " des sens, puisque ce n'est qu'en suivant la nature que la raison - qui est un produit touche l'être véritable. Et l' « art difficile " du « jeune instituteur» est «de tout faire en ne faisant rien », car la «leçon" doit «venir de la chose même $^{27}$ ". Par l'éducation, la conscience humaine accomplit son odyssée ; d'abord sentiment du nécersaire et, partant, de l'extériorité comme telle, de la dépendance et de l'obstacle, elle traverse au mitan le large terrain de l'utile (où le tout est de ne pas s'encombrer et se «fausser » de représentations imaginaires), pour s'apercevoir finalement comme " amour du bon» (" voix de l'âme », «instinct divin»). Elle va du physique au métaphysique, d'une sensibilité d'assujettissement à une sensibilité ontologiquement magnifiée par l'harmonie ${ }^{28}$. L'itinéraire de la conscience éduquée aborde successivement les deux "phénomènes » d'une même Nature : l'ordre et la justice. Le «maître »-c'est-à-dire celui qui «montre » - ne représente pas, mais plutôt aide à voir, assiste (à) la présentation ${ }^{29}$ de la Nature, dans l'aridité du « il

de la science prétendue est ce qui mène d'abord à rentrer dans soi et à se confier au bon sens, cette raison commune qui est autre chose et plus que la raison de tous...

24. «Je ne mets des vérités dans sa tête que pour le garantir des erreurs qu'il apprendrait à leur place », op. cit., Livre II, p. 435.

25. Ibid., Livre IV, p. 639.

26. Ibid., Livre IV, p. 359

27. Ibid., Livre II, p. 362.

28. C'est le $2^{e}$ article de foi du Vicuire savoyard : le « concert», l' « harmonie »: « à quels yeux non prévenus l'ordre sensible de l'univers n'annonce-t-il pas une suprêrne intelligence? $\gg$ Ibid., Livre IV, p. 579.

29. Il faudrait d'ailleurs, sur ce premier paradoxe ( ( faire en ne faisant rien »), en greffer un second qui paraît en prendre le contre-pied puisqu'il va, curieusement chez Rousseau, jusqu'à réhabiliter l'artifice et presque à embaucher le " supplément »... Émile, qui se termine en somme par une méditation sur le goât dont on ne finirait pas de dire la profondeur et la subtilité ensemble, contient une apologie inattendue des signes et des marques. « La seule raison, écrit Rousseau, n'est point active ; elle retient quelquefois, rarement elle excite, et jamais elle n'a rien fait de grand». I bid., p. 654. Les « âmes fortes» comprennent et parlent la 
faut " comme dans l'effusion du « je dois ». Comme la figure Rousseau, l'éducation est le procès médié par quoi la médiation se dénie en faveur d'un immédiat. Qu'il faille un maître est sûr indice de la condition « sensible " et physique de l'homme ; qu'il ne poursuive, s'il est ce qu'il doit être, que son propre abolissement est signe d'une vocation métaphysique. Le " maitre » est cette corde très sensible, tendue entre le nécessaire et le bon, c'est-à-dire reliant la nature à elle-même. La méthode suit d'autant mieux le droit chemin qu'elle cultive son «négatif » (comme dit Rousseau), ou, pour le dire d'un énoncé trivial, qu'elle « ne se met pas en avant ».

\section{LA PHIL DSOPHIE}

En épigraphe à la troisième partie de Vérité et Méthode ${ }^{30}$, intitulée «Inflexion ontologique de l'herméneutique : sous la conduite du langage », $\mathrm{H}$. G. Gadamer place l'énoncé de F. Schleiermacher : «Alles Vorauszusetzende in der Hermeneutik ist nur Sprache » (Tout ce qu'il est besoin de présupposer dans l'herméneutique, c'est la langue). La méthode est si peu extérieure à la vérité, qu'elle se donne, au fond, à comprendre du pré-compris. Toute compréhension intra-méthodique se présuppose elle-même, c'est-à-dire se donne comme son propre commentaire. Dans un autre texte des Kleine Schriften I, Was ist Wahrheit?, Gadamer décrit l'énonciation (Aussage) comme motivée (motiviert) : «Jede Aussage hat Voraussetzungen, die sie nicht aussag ${ }^{31}$. » Toute énonciation a des présupposés qu'elle n'énonce pas. C'est ce qui permet à Gadamer de constituer l'énoncé, quel qu'il soit comme «essentiellement réponse » (« Der Primat der Frage gegenüber der Aussage bedeutet aber, dass die Aussage wesenhaft Antwort ist "). De là découle encore que seul prend la mesure de la vérité d'un énoncé ( « die Wahrheit einer Aussage wirklich ermessen $»$ ), celui qui pense-avec (mitdenkt) les présupposés. La vérité fait donc corps avec le jeu de la question et de la réponse dont le lieu est le langage et la

"langue des signes ". C'est qu'il faut toujours « montrer", aussi au sens de " la montre " pascalienne. Tout se passe comme si « l'objet qu'on expose aux yeux » avait deux valeurs, à la fois contradictoires et intriquées : disons d'excitation primaire et d'ancrage définitif ; comme s'il fallait passer par le torve de l'artifice pour forcer l'attention au plus solicle. «On n'a de prise sur les passions que par les passions". Ibid., p. 654. Le goât, justement, sollicite prudemment l'imagination et alimente les passions, dont il utilise l'énergie "monstrative " et l'homéopathie et que, pour ainsi parler, il sublime. Comme toujours, c'est moins la vérité qui compte que l'absence d'erreurs catastrophiques...

30. Éd. du Seuil, coll. «L'ordre philosophique », 1976, p. 227.

31. Éd. J. C. B. Mohr (Paul Siebeck), Tübingen, 1967, p. 54. À noter que Renan, déjà, s'appuyant sur W. de Humboldt, remarquait, au chap. XV de l'Avenir de la science : * Ce qu'on ne peut trop répéter, c'est que, par les langues, nous touchons le primitif " (R. Laffont, Bouquins, p. 260). Si l'on ne colle pas d'avance à l'auteur l'épithète « scientiste», on observe que le texte renanien est bien loin de " chosifier » ce « primitif » qui ouvre, au contraire, aux sciences de l'homme tout leur espace interprétatif et méthodologique. 
méthode consiste à renvoyer, en quelque sorte, les énoncés à la question dont ils sont la réponse. La méthode, aussi bien, est ensemble commentaire et questionnement. Ou plutôt : le commentaire c'est la question instruite en discours.

Cette structure, si notre intuition est juste, ne vaut pas seulement de la pensée heideggérienne prolongée par l'herméneutique gadamérienne. Elle détermine toute méthode comme pensée anticipante dans le champ de la philosophie. E. Levinas, après avoir posé qu'une «méthode n'est jamais un simple instrument fabriqué pour explorer n'importe quel domaine du réel ", et qu' " il faut avoir une vue anticipée du "sens" de l'être qu' on aborde", observe que « Husserl, comme Bergson, a eu l'intuition de sa philosophie avant de faire une philosophie de l'intuition ${ }^{32}{ }^{\prime}$. Et si l'on suit Bergson, justement, dans le texte fameux sur l' “ intuition philosophique ${ }^{33}$ », on doit tenir que toute grande philosophie s'offre en somme comme le commentaire de son principe. Dès lors, la vérité est indissociable de la méthode ; elle est «unterwegs". Après tout, la Critique de la raison pure s'ouvre et s'achève par des considérations réflexives sur la méthode, identifiée avec "la route critique ", devenue « voie royale ». En tant qu'elle mesure ses énoncés au principe qui déclenche la question et fomente le discours, la méthode, dans sa récurrence authentifiante, désigne la pensée elle-même comme anticipation, c'est-à-dire comme « chemin » ou comme tâche (Aufgabe). C'est le chemin obligé ; non seulement au sens où il n'y en a pas d'autres, mais aussi, à la lettre, parce que cette voie à suivre, à l'exclusion de toute autre, est éprouvée comme la tâche et la responsabilité de la pensée.

Si l'on demandait alors où se trouve la différence entre les méthodes scientifiques et la méthode en philosophie, peut-être, tenu de faire court, pourrait-on simplement dire : la différence réside dans le plus ou moins grand cas qui est fait du langage. Parce que la philosophie ne saurait "comprendre " l'Etre sans se comprendre elle-même, c'est-à-dire sans se réfléchir tout entière dans la méthode (ce en quoi, je le répète, elle est son propre commentaire), elle n'est pas seulement, comme le voulait Kant, le " génie de la raison »; elle est aussi celui de la langue. Celle-ci, en dernière analyse, est son seul modèle légitime.

Nous n'avons pas la prétention, dans les limites de la présente interrogation, de traiter sérieusement de la différence des méthodes scientifique et philosophique, fût-ce en bornant la comparaison aux seules sciences humaines. Une telle confrontation impliquerait sans doute un examen sérieux du concept de modèle et de sa polysémie. Nous nous contenterons de quelques remarques

32. Emmanuel Levinas, Théorie de l'intuition dans la phénoménologie de Husserl, Paris, Vrin, 1963, p. 11-12.

33. Emmanuel Levinas, La Pensée et le Mouvant, Éd. du Centenaire, Paris, PUF, p. 13-45. 
problématiques à valeur d' indices. Lorsque Durkheim ${ }^{34}$, par exemple, recherche les critères du " fait social ", il rabat délibérément la connaissance sur la reconnaissance, l'identité sur l'identification ; si la " première règle et la plus fondamentale est de considérer les faits sociaux comme des choses", cela ne signifie pas que les faits sociaux sont des «choses", mais bien qu'il faut les regarder comme s'ils l'étaient. C'est de la même façon que procède LéviStrauss dans les Structures élémentaires de la parenté ${ }^{35}$, lorsque, constituant la prohibition de l'inceste comme la suture entre les ordres exclusifs de la nature et de la culture, chacun défini par un critère (respectivement l'universel et la règle), il substitue volontairement à une illusoire " analyse réelle " une «analyse idéale ». Ainsi encore, Paul Veyne ${ }^{36}$, tirant la leçon de Marc Bloch ( " un livre d'histoire est la réponse à un problème d'histoire »), fait apparaître que la condition de possibilité de tout discours d'histoire est le choix préalable d'une « intrigue ", c'est-à-dire d'un angle de vue sous le rapport duquel du matériel historique pourra être traité et prendre forme de discours. On retrouve, certes, dans ces attitudes, bien des traits que nous avons dégagés plus haut, en particulier l'importance décisoire du principe. On remarque toutefois que ce principe est un modele et qu'il ne conditionne un discours qu'à lui assigner en même temps des bornes strictes et en le subordonnant à un " comme si » insuppressible : la " manière de dire » s'impose au « dire » dans ce qu'elle a d'oblique et c'est même parce qu'elle est obliqua oratio que la méthode est opératoire. Ayant circonscrit un champ, elle sait y reconnaître des " objets " et des relations. Elle est donc bien un langage, puisqu'elle tient à une "manière de dire "; mais ce langage, foncteur de réalités déterminées, obstrue en même temps $l e$ langage lui-même. On dirait que la méthode scientifique est "modélisante", alors que la méthode philosophique est métaphorique $^{37}$. Si la philosophie est commentaire ${ }^{38}$ de son principe, une discipline scientifique gage sa vertu explicative sur le caractère tacite du principe. Le

34. Émile Durkheim, Les Règles de la méthode sociologique, Paris, PUF, p. 11 et 15.

35. Claude Lévi-Strauss, Structure élémentaire de la parenté, Paris, Ed. Mouton, 1967, p. 9-10.

36. Paul Vegne, Comment on écrit l'histoire ? Paris, Seuil, 1971.

37. Je ne peux que renvoyer à la Septième étude de La Métaphore vive de Paul Ricour (éd. du Seuil, 1975), intitulée « Métaphore et référence ». On y trouvera en particulier des analyses très précises sur les modèles (p. 302 et $s q$.). La thèse soutenue par l'auteur, en général, est que « la suspension de la référence, au sens défini par les normes du discours descriptif, est la condition négative pour que soit dégagé un mode plus fondamental de référence $\gg$ (p. 288).

38. J'emprunte à Michel Foucault ce beau portrait du commentaire : « Le moutonnement indéfini des commentaires est travaillé de l'intérieur par le rêve d'une répétition masquée : à son horizon, il n'y a peut-être rien d'autre que ce qui était à son point de départ, la simple récitation. Le commentaire conjure le hasard du discours en lui faisant la part : il permet bien de dire autre chose que le texte même, mais à condition que ce soit ce texte même qui soit dit et en quelque sorte accompli. » in L'Ordre du discours, Paris, Gallimard, 1971, p. 27-28. 
commentaire ancre la question à même la langue; son idéal est la répétition de l'Être-un ; l'explication, discours indirect, modèle image (fût-ce éminemment intellectualisée), procède par problèmes; son idéal est la totalisation des existants, quel que soit leur mode. La science est discours de sa méthode et par sa méthode ; la philosophie est la méthode du discours.

J'ai commencé par Descartes ; je veux finir par lui. Plus précisément par le modèle ambigü que les Méditations représentent aux yeux d'un Husserl prononçant en Sorbonne, fin février 1929, les fameuses conférences qui deviendront les Méditations cartésiennes.

En même temps que le philosophe allemand place son entreprise de refondation sous l'invocation du «plus grand penseur de la France » (« nous allons essayer de méditer à la manière cartésienne "), jusqu'à bien vouloir, d'abord, regarder la "philosophie transcendantale " comme un « néocartésianisme $^{3 y} »$, il adresse cependant à Descartes, et dès la Première Méditation, la critique suivante : « Descartes lui-même s'était donné d'avance un idéal scientifique, celui de la géométrie, ou, plus exactement, de la physique mathématique. Cet idéal a exercé pendant des siècles une influence néfaste. Du fait qu'il a été adopté par Descartes sans critique préalable, ses Méditations se ressentent aussi. Il semblait naturel à Descartes que la science universelle dût avoir la forme d'un système déductif, système dont tout l'édifice reposerait ordine geometrico sur un fondement axiomatique servant de base absolue à la déduction. L'axiome de la certitude absolue du moi et de ses principes axiomatiques innés joue chez Descartes, par rapport à la science universelle, un rôle analogue à celui des axiomes géométriques en géométrie. Mais le fondement est encore plus profond ici qu'en géométrie et est appelé à constituer le dernier fondement de la science géométrique elle-même ${ }^{40}$ ». Je ne me propose pas d'entrer dans le fond de ce reproche ; ce qui m'intéresse, c'est son genre. Ce que dit Husserl, c'est qu'un fondement-écran s'est interposé, bouchant l'accès au fond ; qu'il a obnubilé l'intention du discours en prenant la forme d'un idéal — autant dire d'un modèle — en abusant l'auteur luimême par sa soi-disant évidence ( il semblait naturel à Descartes"). Que Descartes, donc, ait, avec ses Méditations, bâti le « prototype du retour philosophique sur soi-même ", Husserl l'en crédite de prime abord ; et c'est même depuis cette base positive qu'il le reprend sur ce qu' on pourrait appeler une défaillance au couur même de la radicalité.

C'est que, aussi réflexif qu'il puisse être, un modèle participe immanquablement d'une " épistémè " datée, qui clôture les mots et les choses, tout simplement à les distribuer ; dans cet arrangement qui fait horizon, phénoménologiquement et « logiquement », passe pour « nature » ce qui est « époque ». De strictement opératoire ou instructif, le modèle glisse à l'idéal, c'est-à-dire à l'absolu d'une forme-canon indépassable; ainsi du more geometrico.

39. Edmund Husserl, Méditations cartésiennes, Paris, Vrin, p. 1.

40. Ibid., p. 6. 
L'analogon devient Logos. Et la méthode, mathématisée, appelle le système, c'est-à-dire une totalisation aveugle à ses axiomes. Si Descartes, selon Husserl a péché par prévention (toujours le : «il semblait naturel ") et par précipitation (l'« idéal scientifique » adopté par Descartes « sans critique préalable »), c'est pour avoir, au fond, gelé la référence, pour en avoir pétrifié le jeu, s'il est vrai que la radicalité du discours philosophique exige, justement, la destruction en cours de route de la référence. Après avoir fermé la porte aux vérités de mauvais aloi, le verrou géométrique eût dû sauter et l'axiomatique s'effacer devant l'anhypothétique. Bref, le langage idéal a obturé l'idéal du langage; la réponse a oublié sa question.

Si le modèle « appartient non à la logique de la preuve, mais à la logique de la découverte ${ }^{41} », s^{\prime}$ il est instrument de rupture et de réforme, il lui convient de se maintenir en balance et comme par provision, de libérer du réel, non de l'occuper. Dans une science, ou plutôt dans une discipline scientifique, la délimitation même de la région et du genre des étants considérés peut prévenir l'arrogance du modèle. Ces garde-fous, par définition, n'existent pas en philosophie et, pour parler comme Kant, il est de la raison pure de s'autodiscipliner ${ }^{42}$. Or, parce qu'aucun modèle ne correspond à l'Être en lui-même, celui-ci, dans sa pure différence, n'est-il pas, plutôt qu'en des représentations durcies et imaginaires, plus à l'abri dans la haute mer du langage, on allait dire dans ses marées qui, justement, font et défont le sens, détruisent pour innover?

J'aimerais à la fin pouvoir réciter mot pour mot l'admirable passage de Philèbe (15-17) qui contient l' «éthique dialectique » de Platon. Socrate affirme à Protarque que « l'identification de un et plusieurs opérée par le langage » est « une propriété impérissable et toujours neuve de nos propos ». La dialectique est « cadeau que les dieux ont fait à l'homme». Mais c'est un bien d'autant plus fragile qu'il s'expose à l'ordinaire des rencontres et des jours. Parler trop vite comme parler habilement le corrompt, puisque ce bien, de soi pudique, vit de la vie publique du langage. D'enthousiasme, les jeunes gens, étonnés de cette sagesse infuse, le maltraitent; et, alors que les dieux nous l'ont donné pour nous permettre " de chercher et d'apprendre, et de nous instruire les uns les autres ", " les doctes du monde d'aujourd'hui font "un" au petit bonheur et "plusieurs" trop vite ou trop lentement, passant immédiatement de l'un à l'infini, tandis que les intermédiaires leur échappent ».

Moins qu'à un idéal de science, la méthode du discours, pour ne pas trahir sa tâche spécifique, se repère à deux injonctions envoyées par le langage

41. Paul Riccur, op. cit, p. 302.

42. Emmanuel Kant, Critique de la raison pure, Théorie transcendantale de la méthode, Discipline de la raison pure. 
lui-même : l'innovation métaphorique et la transparence éthique, l'une et l'autre étant corrélatives. Dans l'un et l'autre cas, en effet, il est dit simplement que le langage ne doit pas boucher la route du langage. À cet égard, des réflexions comme celles que propose un Jürgen Habermas dans Connaissance et intérêt et, plus encore, dans la Théorie de l'agir communicationnel me paraissent plus contribuer à l' " éthique » de la méthode - ne serait-ce que par le rajeunissement du concept d' « activité communicationnelle », dialogique - , la raison ne devant être ni confisquée par l'instrumentalité doctorale ni dévoyée par l' " enthousiasme " médiatique, qu'à sa " métaphorisation ".

Mais ce serait, ici où il faut que je m'arrête, le commencement d'une autre recherche de méthode, qui, directement, interrogerait le lien entre l'éthique et le fictif.

Université de Provence 